Research Paper

\title{
Usefulness of Bronchoscopic Rebiopsy of Non-Small Cell Lung Cancer with Acquired Resistance to Epidermal Growth Factor Receptor-Tyrosine Kinase Inhibitor
}

Eun Kyong Goag1 ${ }^{1 *}$, Jung Mo Lee ${ }^{1 *}$, Kyung Soo Chung1 ${ }^{1}$, Song Yee Kim ${ }^{1}$, Ah Young Leem ${ }^{1}$, Joo Han Song ${ }^{1}$, Ji

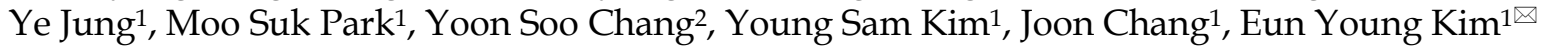

1. Division of Pulmonology, Department of Internal Medicine, Severance Hospital, Yonsei University College of Medicine, Seoul, Republic of Korea

2. Division of Pulmonology, Department of Internal Medicine, Gangnam Severance Hospital, Yonsei University College of Medicine, Seoul, Republic of Korea

*Contributed equally

$\triangle$ Corresponding author: Eun Young Kim, MD, PhD, Division of Pulmonology, Department of Internal Medicine, Yonsei University College of Medicine, 50-1 Yonsei-ro, Seodaemun-gu, Seoul 03722, Republic of Korea. Phone: 82-2-2228-2267; Fax: 82-2-393-6884; E-mail: narae97@yuhs.ac

(C) Ivyspring International Publisher. This is an open access article distributed under the terms of the Creative Commons Attribution (CC BY-NC) license (https://creativecommons.org/licenses/by-nc/4.0/). See http://ivyspring.com/terms for full terms and conditions.

Received: 2017.06.27; Accepted: 2018.02.02; Published: 2018.03.02

\begin{abstract}
Background: Approximately $50 \%$ of non-small cell lung cancer (NSCLC) patients with acquired resistance to EGFR-TKI harbor the EGFR mutation T790M. The recent development and wide use of third-generation EGFR-TKIs targeting T790M-mutant NSCLCs have increased the importance of rebiopsy after EGFR-TKI failure. We aimed to investigate the advantages of flexible bronchoscopy as a rebiopsy method and the prevalence of and factors affecting the T790M mutation after EGFR-TKI failure.

Methods: We investigated 139 patients who had undergone bronchoscopic rebiopsy and endobronchial ultrasound-guided transbronchial needle aspiration (EBUS-TBNA) between Sep 2014 and Jul 2016.

Results: Among the 139 patients, bronchoscopic rebiopsy yielded successful pathological diagnoses in 102 (73.4\%). Among them, 41 patients with EGFR-mutant lung adenocarcinoma and EGFR-TKI progression were selected for an investigation of T790M mutation prevalence at rebiopsy. The initial EGFR mutations were exon $19 \mathrm{del}(56.1 \%)$, L858R or L861Q (34.1\%), and others $(9.8 \%)$. The most common rebiopsy method was transbronchial lung biopsy $(41.5 \%)$, followed by EBUS-TBNA (26.8\%) and endobronchial biopsy (19.5\%). The median interval to T790M emergence was the longest among cases with exon 19 deletion (14.1 months), followed by exon 21 L858R or L861Q (11.3 months) and other rare EGFR mutations (2.9 months). The T790M mutation was identified in 18 (43.9\%) patients, and exon 19 del was the most significant factor affecting T790M mutation development (hazard ratio: $6.875, P=0.014$ ).

Conclusions: Bronchoscopy was more useful than other rebiopsy approaches. The T790M emergence rate was highest in cases with exon 19 deletion, likely as a consequence of long-term EGFR-TKI exposure.
\end{abstract}

Key words: rebiopsy, T790M, epidermal growth factor receptor-tyrosine kinase inhibitor (EGFR-TKI), non-small cell lung cancer (NSCLC)

\section{Introduction}

Recently, our understanding of non-small cell lung cancer (NSCLC), the leading cause of cancer-related mortality worldwide[1], and its treatment has evolved dramatically, largely due to a better understanding of the underlying genomic alterations. Currently, mutations in epidermal growth factor receptor $(E G F R)[2,3]$ are the best-described of these alterations, and targeted EGFR-tyrosine kinase inhibitors (EGFR-TKI) have yielded dramatic responses with reduced toxicity in patients with EGFR-mutant NSCLC[4]. However, virtually all patients with EGFR-mutant NSCLC benefit from 
EGFR-TKI treatment for less than 1 year, after which drug resistance develops[5].

Several resistance mechanisms have been identified. Of these, the EGFR c.2369C >T (T790M) mutation within exon 20 is most prevalent, accounting for approximately $50 \%$ of EGFR-mutated tumors with acquired erlotinib or gefitinib resistance[6]. Recently developed third-generation EGFR-TKIs that target T790M are effective against NSCLCs harboring this mutation and are now widely used in clinical settings[7]. These new treatment options have increased the importance of rebiopsy for treatment guidance in patients with progressive disease and a previous history of EGFR-TKI therapy. However, several factors limit the feasibility of rebiopsy and molecular analysis, including the amount of required material, access to the tumor site, and the invasiveness of sampling methods, particularly surgical rebiopsy[8-10].

Therefore, we aimed to investigate the advantages of rebiopsy via flexible bronchoscopy. This technique is commonly used, minimally invasive, and can collect abundant tissue samples for histological and molecular analyses. Furthermore, we aimed to investigate the prevalence of T790M mutation after EGFR-TKI failure, as well as factors affecting the T790M mutation rate.

\section{Methods}

\section{Study design and patients}

This was a single center, observational, retrospective study conducted at Severance Hospital (Seoul, Republic of Korea), a university-affiliated tertiary care referral hospital. The study protocol was approved by the Severance hospital institutional review board (IRB No. 4-2016-1100).

We investigated 140 patients who had undergone rebiopsy via flexible bronchoscopy (endobronchial biopsy and transbronchial lung biopsy (TBLB)) and endobronchial ultrasound-guided transbronchial needle aspiration (EBUS-TBNA) between September 2014 and July 2016. After excluding 1 patient with a de novo T790M mutation, 139 patients remained. We investigated the practical success rate (number of patients in which tumor cells were detected/total number of rebiopsy cases performed $\times 100$ as defined elsewhere[11]) and the procedure-related complication rate.

We additionally selected a subgroup of 41 patients to investigate the T790M mutation prevalence and determine the factors affecting T790M detection. The selected patients had pathologically confirmed lung adenocarcinoma, known EGFR mutations in pre-EGFR-TKI treatment tumor specimens, a history of EGFR-TKI therapy, and available rebiopsy tumor specimens for EGFR mutation status assessment (Figure 1).

\section{EGFR mutation analysis}

Genomic DNA was purified from formalin-fixed, paraffin-embedded sections of tumors using the ReliaPrep ${ }^{\text {TM }}$ FFPE gDNA Miniprep system (Promega, Madison, WI, USA). EGFR mutations were detected by the Peptide Nucleic Acid (PNA) real-time PCR assay using PNA Clamp ${ }^{\mathrm{TM}}$ EGFR mutation detection kit (Panagene, Daejeon, Republic of Korea).

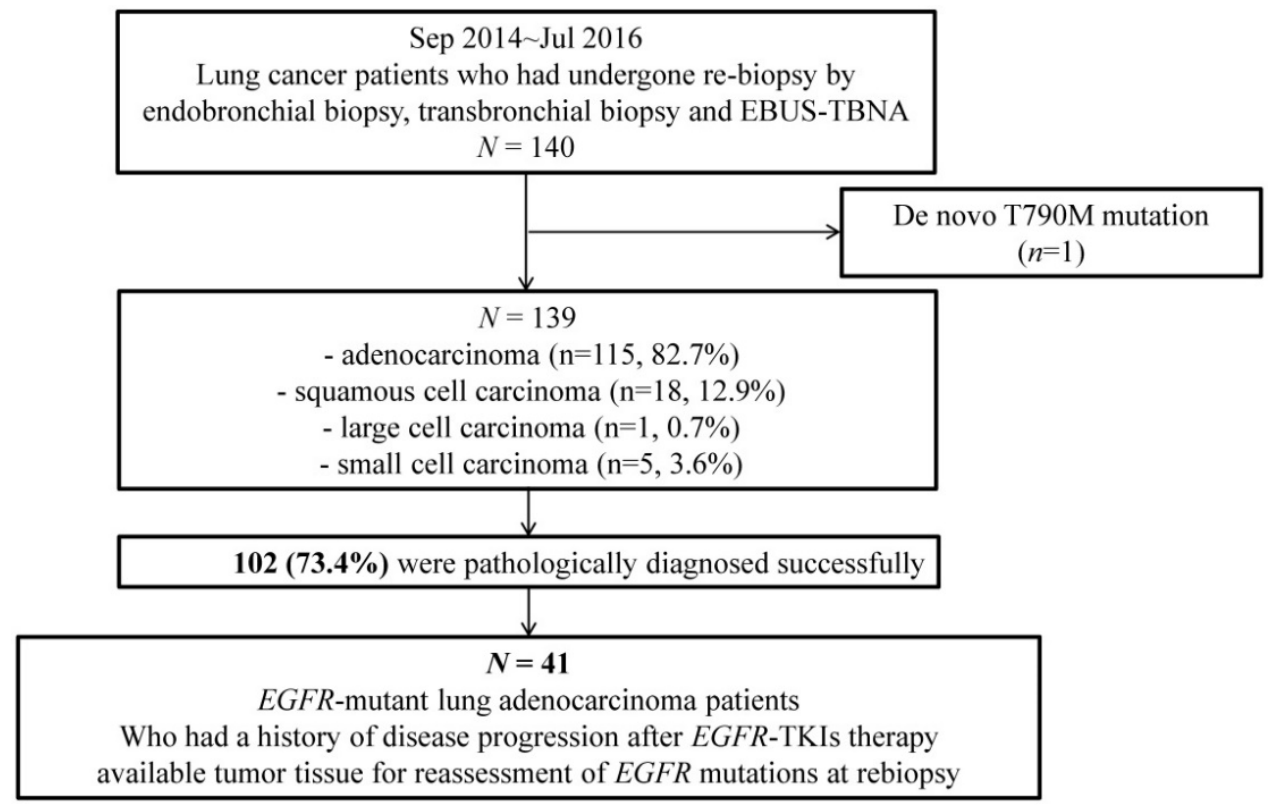

Figure 1. Study design and inclusion and exclusion criteria. 


\section{Data collection and study endpoints}

The analyzed clinical data included age, sex, smoking status, performance status (Eastern Cooperative Oncology Group; ECOG), EGFR mutation status, EGFR-TKI treatment status and response, progression-free survival (PFS), and rebiopsy condition and method. These data were collected retrospectively from medical records. PFS was calculated as the interval between the date of treatment initiation and the date of progression on EGFR-TKI treatment. Unidimensional measurement, as defined by the Response Evaluation Criteria in Solid Tumors (Version 1.1), was implemented in this study[12]. The primary endpoints were the practical success rate of rebiopsy by flexible bronchoscopy and the prevalence of T790M mutations among post-EGFR-TKI rebiopsies. The secondary endpoints were the factors influencing T790M mutation detection.

\section{Statistical analysis}

A univariate analysis ( $X^{2}$ test or Fisher's exact test) of the T790M mutation frequency was conducted to evaluate the effects of clinical factors. We considered two aspects regarding rebiopsy timing: 1) whether patients had received treatment other than EGFR-TKI between the first EGFR-TKI progression and rebiopsy; those who had received such treatment were defined as "with interval from first EGFR-TKI progression", and vice versa. 2) Whether patients continued to receive EGFR-TKI treatment within 1 month before rebiopsy; those who continued receiving such treatment were defined as "with EGFR-TKI treatment at rebiopsy", and vice versa. The effects of clinical factors on the T790M mutation rate were evaluated using a multiple logistic regression model. A two-sided $P$ value of $<0.05$ was considered to indicate statistical significance. Statistical analyses were performed using the SPSS software package (ver. 20.0; SPSS. Inc., Chicago, IL, USA).

\section{Results}

\section{Patient demographics and features of bronchoscopic rebiopsy}

Regarding histology, of the 139 enrolled patients, $115(82.7 \%), 18(12.9 \%), 1(0.7 \%)$, and $5(3.6 \%)$ patients had adenocarcinoma, squamous cell carcinoma, large cell carcinoma, and small cell carcinoma, respectively. We successfully obtained the tumor tissues and pathologic diagnoses of 102 patients, for an overall practical success rate of $73.4 \%$. Only 1 patient developed a complication after rebiopsy. Although he experienced a bleeding event during transbronchial lung rebiopsy, he was discharged without any problems.

Next, we selected 41 patients for the with EGFR mutation status subgroup analysis, as described in the Methods (Figure 1). The baseline characteristics of this subgroup, including the EGFR-TKI treatment and rebiopsy statuses, are shown in Table 1 . The median patient age in the subgroup was 61 years (range: 26-80 years). Twenty-six patients $(63.4 \%)$ were female, and 31 (75.6\%) were never-smokers. Exon 19 deletion was the most common type of EGFR mutation $(56.1 \%)$, followed by exon 21 L858R or L861Q (34.1\%). Another 4 patients (9.8\%) harbored other rare EGFR mutations, including Exon 18 G719X, Exon 20 S768I, and two E20 insertion/duplication mutations. The median duration of EGFR-TKI treatment was 10 months (range: $0.5-35$ months).

Table 1. Demographic data and characteristics of EGFR mutant adenocarcinoma patients.

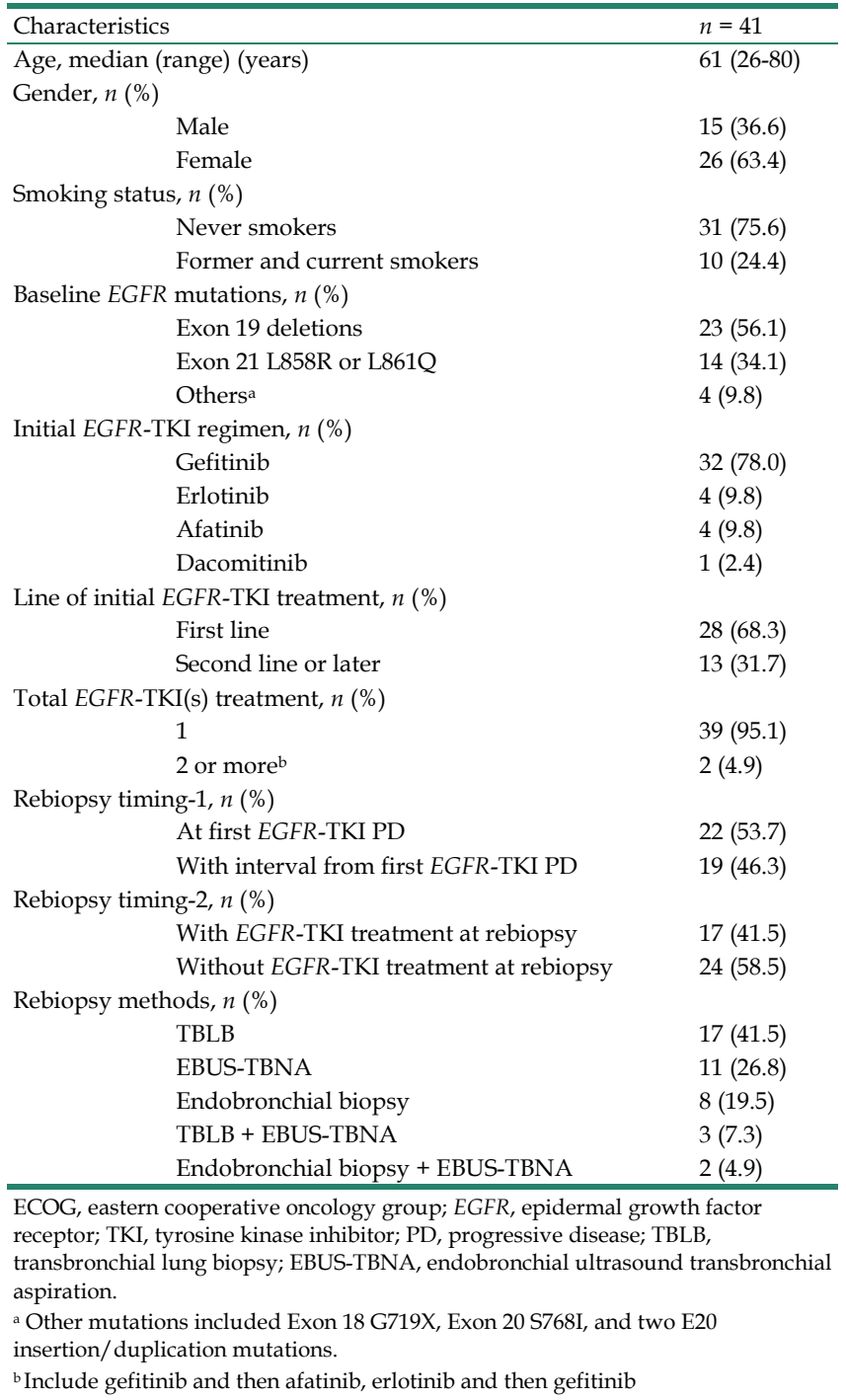


Gefitinib was the most commonly used EGFR-TKI (78.0\%). Most subgroup patients (68.3\%) received EGFR-TKI as the first line of therapy, and the majority $(95.1 \%)$ received only one EGFR-TKI. Rebiopsy was performed at the time of the first EGFR-TKI progression in 22 patients $(53.7 \%)$, and within a median 2-month (range: 2-34 months) interval after the first EGFR-TKI progression in 19 patients $(46.3 \%)$. At the time of rebiopsy, 17 patients $(41.5 \%)$ continued receiving EGFR-TKI treatment, whereas the other 24 ceased EGFR-TKI use. Regarding the rebiopsy method, $17(41.5 \%), 11$ (26.8\%), 8 (19.5\%), 3 (7.3\%), and 2 (4.9\%) patients underwent TBLB, EBUS-TBNA, endobronchial biopsy, TBLB with EBUS-TBNA, and endobronchial biopsy with EBUS-TBNA, respectively.

\section{EGFR T790M mutation status at rebiopsy}

Eighteen (43.9\%) of the rebiopsy specimens from the 41 patients with EGFR mutant adenocarcinoma were found to be positive for the T790M mutation. All 18 patients acquired the T790M mutation along with original EGFR mutations; in other words, none had newly acquired the T790M mutation without an original EGFR mutation. Of the remaining 23 patients, $18(43.9 \%)$ had only the original EGFR mutation, and 5 patients $(12.2 \%)$ had lost the activating EGFR mutation at the time of rebiopsy. Next, we investigated the frequency of T790M among the rebiopsies and observed differences in the rate of
EGFR T90M emergence according to the initial EGFR mutation status. The T790M emergence rate was highest among cases with exon 19 deletion (65\%), followed by exon 21 L858R or L861Q mutation $(21.5 \%)$, but was not associated with other rare EGFR mutations $(P=0.002)$. The median interval to T790M emergence was the longest among cases with exon 19 deletion (14.1 months), followed by exon 21 L858R or L861Q (11.3 months) and other rare EGFR mutations (2.9 months). Loss of the activating EGFR mutation was not observed in Exon 19 deletions (Figure 2).

\section{Factors affecting the emergence of EGFR T790M mutation}

Next, univariate analyses of the patients' characteristics and conditions related to EGFR-TKI treatment were performed to identify factors affecting the emergence of the T790M mutation (Table 2). Age, sex, and smoking status were not found to associate significantly with the emergence of the T790M mutation. Patients with exon 19 deletions at the initial biopsy had a higher T790M mutation rate than did those with exon 21 L858R or L861Q mutations and others $(P=0.002)$. Of note, EGFR-TKI-treated patients who exhibited better responses (partial response vs. stable or progressive disease) to the initial treatment tended to have a higher T790M mutation rate, although this was not statistically significant $(P=$ 0.075).

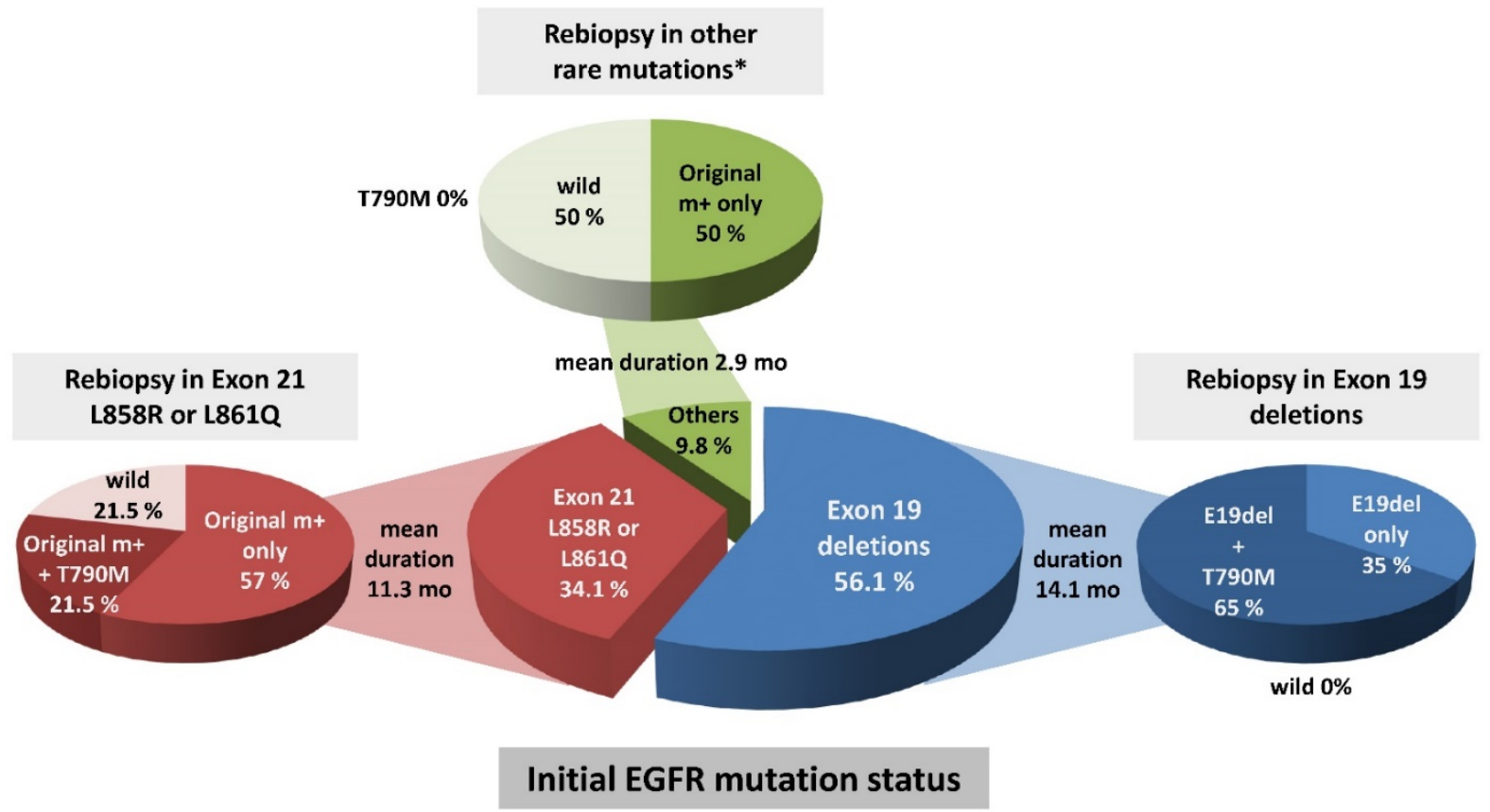

Figure 2. Comparison of EGFR mutation status at initial biopsy and rebiopsy. T790M was most prevalent in initial Exon 19 deletions compared to Exon 21 L858R or $861 \mathrm{Q}$ mutations and other rare mutations ( $P=0.002$ by $\chi^{2}$ test). Mean duration of disease progression after EGFR-TKI treatment was 14.1 months in initial Exon 19 deletions, 11.3 months in initial Exon 21 L858R or $861 \mathrm{Q}$ mutations, and 2.9 months in initial other rare EGFR mutations ( $P=0.028$ by one-way ANOVA test). *Other rare mutations included Exon 18 G719X, Exon 20 S768I, and two E20 insertion/duplication mutations. 
with progressive cancer and a history of EGFR-TKI treatment has increased with the development and use of third-generation EGFR-TKIs, which inhibit both activating EGFR and resistant T790M mutations while sparing wild-type EGFR[15, 16]. Therefore, we investigated the usefulness of bronchoscopy for rebiopsy in these patients.

We observed similar rates of practical rebiopsy success with bronchoscopy between our study and the reports of other studies of bronchoscopic rebiopsy. Specifically, bronchoscopy was found to be the most useful rebiopsy tool, as it yielded a high success rate and low incidence of complications. In most rebiopsy cases, a mutation analysis was performed to determine the therapeutic agent for lung adenocarcinoma. Because lung adenocarcinomas arise mostly in the lung parenchyma, rather than the endobronchus, rebiopsies are performed via bronchoscopy, percutaneous needle aspiration biopsy (PCNAB), or surgical resection, especially for peripheral lesions. Although percutaneous needle aspiration is highly successful when performed under imaging guidance, this technique does not collect sufficient tumor cells for a multiple mutation analysis and is associated with a relatively high risk of pneumothorax. In a rebiopsy study using CT-guided PCNAB, Yoon et al. reported an $80 \%$ rate of success regarding the collection of sufficient specimens for mutational analysis, but observed that $14 \%$ of patients suffered biopsy-related complications[17]. In another rebiopsy study using CT-guided core biopsy, the rate of adequate specimen acquisition was $90 \%$, although $20 \%$ of patients experienced biopsy-related complications[18].

Surgical resection is contraindicated in patients with a poor performance status. However, transbronchial lung biopsy via bronchoscopy yields a larger amount of tumor cells compared to PCNAB, and confers a relatively low risk of pneumothorax. Although previous rebiopsy studies have reported lower success rates for transbronchial lung biopsy via bronchoscopy relative to those for PCNAB, the use of modalities such as electromagnetic navigation bronchoscopy, radial EBUS-GS (guide sheath) and virtual bronchoscopic navigation can enhance the success rate of bronchoscopic rebiopsy, particularly for small peripheral lung lesions. Notably, a rebiopsy study using EBUS-GS reported a rebiopsy success rate of $79.2 \%$, similar to that reported in PCNAB studies, with no severe complications[19]. Another study using EBUS-GS for the diagnosis of peripheral pulmonary lesions reported a pneumothorax occurrence rate of $<1 \%[20]$. In addition, newest approaches to complement the weakness of bronchoscopic diagnosis of lung nodules have been introduced. TBLB using ultrathin bronchoscope showed higher diagnostic yield than conventional bronchoscope[21, 22]. Conebeam-CT guided TBLB showed twofold higher results than conventional methods for the diagnosis of peripheral nodules less than 20mm[23]. In addition, bronchoscopic trahnsparenchymal nodule access (BTPNA) has been attempted for nodules without "bronchus sign" (airway directly reading to nodules)[24, 25]. If these newest techniques are applied to rebiopsy, the diagnostic yield of bronchoscopic rebiopsy can be further improved and the role of bronchoscopy will be even greater in the rebiopsy area.

In addition, transbronchial biopsy of the primary tumor and EBUS-TBNA of the metastatic mediastinal LN (i.e., multiple sites) can be performed simultaneously, which presents an advantage in terms of overcoming spatial intrathoracic T790M heterogeneity and enabling a careful analysis of the T790M status. In fact, 1 case in the present study did not harbor T790M in the primary lung tumor, whereas EBUS-TBNA revealed this mutation in the mediastinal LN. The patient was subsequently treated with a third-generation EGFR-TKI. Another study reported a higher $T 790 \mathrm{M}$ detection rate for the rebiopsy of metastatic lung lesions, compared with biopsy of primary tumors[18]. Similarly, our study found a higher $7790 \mathrm{M}$ detection rate in the metastatic mediastinal lymph nodes $(63.6 \%)$ than in primary tumors (36.7\%), although this difference did not reach statistical significance. Although reports suggest that T790M detection does not correlate with the biopsy location[26, 27], biopsy of both the primary tumor and metastatic lung lesion could increase the detection rate. In summary, rebiopsy via bronchoscopy is minimally invasive and safely allows the simultaneous collection of sufficient amounts of tumor cells from multiple sites during a single procedure. Therefore, bronchoscopic rebiopsy is more appropriate than PCNAB as a means to overcome the considerable tumor heterogeneity observed in EGFR-TKI-treated patients with EGFR-mutant lung cancer.

Liquid biopsy has recently been introduced for detecting the T790M mutation. This analysis of circulating tumor cells and cell-free circulating nucleic acids enables a more comprehensive assessment of tumor heterogeneity, identification of resistance mechanisms, and real-time monitoring of treatment responses in solid tumors[28]. However, the concordance rates of liquid biopsy with conventional biopsy still vary widely (50-83\%) [29-32]. Given this uncertain reproducibility, liquid biopsy is not yet widely used in lung cancer patients in daily practice[33]. However, this is expected to change with 
the development of more sophisticated technologies. In addition, cell-free circulating nucleic acids can be obtained from sputum, pleural fluid, and bronchoalveolar lavage fluid (BALF), as well as plasma. Compared to plasma, BALF is expected to better reflect the tumor DNA status when evaluated using a tumor-derived extracellular vesicle isolation method[34], which is likely to increase in popularity in the future. Especially for the T790M detection by liquid biopsy, plasma EGFR assay was approved by FDA as a blood-based companion diagnostic for the osimertinib in 2016. Recent study shows that sensitivity of plasma T790M detection was $70 \%$ and an alternate paradigm was suggested that plasma genotyping for T790M would be used as a screening test before tissue biopsy[35]. But in the cases of plasma T790M negative, plasma cannot fully replace tissue biopsy because the false-negative rate is high up to $30 \%$. So careful interpretation is needed in plasma T790M negative cases, and tissue biopsy must be performed to complement the plasma test.

We additionally investigated the frequency of EGFR T790M among rebiopsies and observed a difference in the $7790 \mathrm{M}$ emergence rate according to the initial EGFR mutation status. As shown in Figure 2 , the T790M emergence rate was highest among cases with exon 19 deletions (65\%), and this group [18] had the longest median interval until T790M emergence (14.1 months). Similarly, in a previous study of Japanese patients, T790M more frequently emerged in patients with initial exon 19 deletions $(55.6 \%)$ than in those with L858R mutations (43.0\%) $(P=0.005)$ [11]. In another Japanese study, the T790M mutation was also more frequent in patients with an exon 19 deletion mutation $(63 \%)$ than in those with a L858R mutation (38\%) $(P=0.035)[36]$. Other previous studies have shown similar trends. We attribute these findings to the tendency of cases involving exon 19 deletion to exhibit the most favorable responses and to receive EGFR-TKI treatment for longer durations, compared to cases with other EGFR mutations. Kuiper et al. reported that patients who developed T790M mutations after TKI therapy (detected via biopsy) had a longer median PFS, compared to T790M-negative patients (14.2 vs. 11.1 months, $P=0.034$ )[37]. Recently, Zou et al. investigated the underlying causes of high T790M expression in cases of exon 19 deletion using computational modeling and molecular dynamics simulations[38]. The authors reported that when compared with exon 19 non-LRE deletions or L858R mutation, a delE746_A750 mutation was associated with a higher probability of acquiring the T790M mutation after EGFR-TKI. Additionally, Zou and colleagues found that delE746_A750 exhibited lower stability around the residue T790M, compared with
delS752_I759 and L858R, suggesting that this might explain the strong expression of T790M in cases of exon 19 deletion mutation. Together, these results suggest an association of initial EGFR mutation status and long-term EGFR-TKI exposure with T790M acquisition.

This study has some limitations. Data were retrospectively analyzed and the number of study subjects was relatively small. However, it has the advantage of reflecting the practice using various type of bronchoscopic rebiopsy including endobronchial biopsy, transbronchial lung biopsy and EBUS-TBNA in the real-world conditions.

\section{Conclusions}

We have demonstrated the usefulness of bronchoscopic rebiopsy over other approaches. Additionally, we observed that the T790M emergence rate was highest among cases with exon 19 deletion, possibly attributable to long-term EGFR-TKI exposure in these cases.

\section{Clinical Practice Points}

- Rebiopsy has become essential for treatment guidance in patients with progressive lung adenocarcinoma and a previous history of EGFR-TKI therapy.

- We assessed the advantages of flexible bronchoscopy as a rebiopsy method and the prevalence of and factors affecting the T790M mutation after EGFR-TKI failure.

- The success rate of bronchoscopic rebiopsy was $73.4 \%$. The most common rebiopsy method was transbronchial lung biopsy (41.5\%), followed by EBUS-TBNA (26.8\%) and endobronchial biopsy $(19.5 \%)$.

- The T790M mutation was identified in 18 (43.9\%) patients. The median interval to T790M emergence was the longest among cases with exon 19 deletion (14.1 months), followed by exon 21 L858R or L861Q (11.3 months) and other rare EGFR mutations (2.9 months).

- The T790M emergence rate was highest in cases with exon 19 deletion (65\%), likely as a consequence of long-term EGFR-TKI exposure.

\section{Acknowledgments}

This study was supported by the Basic Science Research Program through the National Research Foundation of Korea (NRF), funded by the Ministry of Science, ICT, and Future Planning (grant No. NRF-2015R1C1A1A02037675 to EY Kim). 


\section{Conflicts of Interest}

No potential conflicts of interest relevant to this article have been reported.

\section{References}

1. Siegel RL, Miller KD, Jemal A. Cancer Statistics, 2017. CA: a cancer journal for clinicians. 2017; 67: 7-30.

2. Lynch TJ, Bell DW, Sordella R, Gurubhagavatula S, Okimoto RA, Brannigan $\mathrm{BW}$, et al. Activating mutations in the epidermal growth factor receptor underlying responsiveness of non-small-cell lung cancer to gefitinib. N Engl J Med. 2004; 350: 2129-39.

3. Paez JG, Janne PA, Lee JC, Tracy S, Greulich H, Gabriel S, et al. EGFR mutations in lung cancer: correlation with clinical response to gefitinib therapy. Science. 2004; 304: 1497-500.

4. Mok TS, Wu YL, Thongprasert S, Yang CH, Chu DT, Saijo N, et al. Gefitinib or carboplatin-paclitaxel in pulmonary adenocarcinoma. N Engl J Med. 2009; 361: 947-57.

5. Rosell R, Moran T, Queralt C, Porta R, Cardenal F, Camps C, et al. Screening for epidermal growth factor receptor mutations in lung cancer. N Engl J Med. 2009; 361: 958-67.

6. Kobayashi S, Boggon TJ, Dayaram T, Janne PA, Kocher O, Meyerson M, et al. EGFR mutation and resistance of non-small-cell lung cancer to gefitinib. N Engl J Med. 2005; 352: 786-92.

7. Janne PA, Yang JC, Kim DW, Planchard D, Ohe Y, Ramalingam SS, et al. AZD9291 in EGFR inhibitor-resistant non-small-cell lung cancer. N Engl J Med. 2015; 372: 1689-99.

8. Chouaid C, Dujon C, Do P, Monnet I, Madroszyk A, Le Caer H, et al. Feasibility and clinical impact of re-biopsy in advanced non small-cell lung cancer: a prospective multicenter study in a real-world setting (GFPC study 12-01). Lung cancer (Amsterdam, Netherlands). 2014; 86: 170-3.

9. Hasegawa T, Sawa T, Futamura Y, Horiba A, Ishiguro T, Marui T, et al. Feasibility of Rebiopsy in Non-Small Cell Lung Cancer Treated with Epidermal Growth Factor Receptor-Tyrosine Kinase Inhibitors. Intern Med. 2015; 54: 1977-80.

10. Kirita K, Izumo T, Matsumoto Y, Hiraishi Y, Tsuchida T. Bronchoscopic Re-biopsy for Mutational Analysis of Non-small Cell Lung Cancer. Lung. 2016; 194: 371-8.

11. Nosaki K, Satouchi M, Kurata T, Yoshida T, Okamoto I, Katakami N, et al. Re-biopsy status among non-small cell lung cancer patients in Japan: A retrospective study. Lung cancer (Amsterdam, Netherlands). 2016; 101: 1-8

12. Eisenhauer EA, Therasse P, Bogaerts J, Schwartz LH, Sargent D, Ford R, et al. New response evaluation criteria in solid tumours: revised RECIST guideline (version 1.1). Eur J Cancer. 2009; 45: 228-47.

13. Huang L, Fu L. Mechanisms of resistance to EGFR tyrosine kinase inhibitors. Acta Pharm Sin B. 2015; 5: 390-401.

14. Tartarone A, Lerose R. Clinical approaches to treat patients with non-small cell lung cancer and epidermal growth factor receptor tyrosine kinase inhibitor acquired resistance. Ther Adv Respir Dis. 2015; 9: 242-50.

15. Steuer CE, Khuri FR, Ramalingam SS. The next generation of epidermal growth factor receptor tyrosine kinase inhibitors in the treatment of lung cancer. Cancer. 2015; 121: E1-6.

16. Cross DA, Ashton SE, Ghiorghiu S, Eberlein C, Nebhan CA, Spitzler PJ, et al. AZD9291, an irreversible EGFR TKI, overcomes T790M-mediated resistance to EGFR inhibitors in lung cancer. Cancer Discov. 2014; 4: 1046-61.

17. Yoon HJ, Lee HY, Lee KS, Choi YL, Ahn MJ, Park K, et al. Repeat biopsy for mutational analysis of non-small cell lung cancers resistant to previous chemotherapy: adequacy and complications. Radiology. 2012; 265: 939-48.

18. Kim H, Chae KJ, Yoon SH, Kim M, Keam B, Kim TM, et al. Repeat biopsy of patients with acquired resistance to EGFR TKIs: implications of biopsy-related factors on T790M mutation detection. European radiology. 2017.

19. Izumo T, Matsumoto Y, Chavez C, Tsuchida T. Re-biopsy by endobronchial ultrasound procedures for mutation analysis of non-small cell lung cancer after EGFR tyrosine kinase inhibitor treatment. BMC pulmonary medicine. 2016; 16: 106.

20. Hayama M, Izumo T, Matsumoto Y, Chavez C, Tsuchida T, Sasada S. Complications with Endobronchial Ultrasound with a Guide Sheath for the Diagnosis of Peripheral Pulmonary Lesions. Respiration; international review of thoracic diseases. 2015; 90: 129-35.

21. Oki M, Saka H, Ando M, Asano F, Kurimoto N, Morita K, et al. Ultrathin Bronchoscopy with Multimodal Devices for Peripheral Pulmonary Lesions. A Randomized Trial. Am J Respir Crit Care Med. 2015; 192: 468-76.

22. Asano F, Shinagawa N, Ishida T, Shindoh J, Anzai M, Tsuzuku A, et al. Virtual bronchoscopic navigation combined with ultrathin bronchoscopy. A randomized clinical trial. Am J Respir Crit Care Med. 2013; 188: 327-33.

23. Hohenforst-Schmidt W, Zarogoulidis P, Vogl T, Turner JF, Browning R, Linsmeier B, et al. Cone Beam Computertomography (CBCT) in Interventional Chest Medicine - High Feasibility for Endobronchial Realtime Navigation. Journal of Cancer. 2014; 5: 231-41.

24. Herth FJ, Eberhardt R, Sterman D, Silvestri GA, Hoffmann H, Shah PL. Bronchoscopic transparenchymal nodule access (BTPNA): first in human trial of a novel procedure for sampling solitary pulmonary nodules. Thorax. 2015; 70: 326-32.

25. Harzheim D, Sterman D, Shah PL, Eberhardt R, Herth FJ. Bronchoscopic Transparenchymal Nodule Access: Feasibility and Safety in an Endoscopic Unit. Respiration; international review of thoracic diseases. 2016; 91: 302-6.

26. Sun JM, Ahn MJ, Choi YL, Ahn JS, Park K. Clinical implications of T790M mutation in patients with acquired resistance to EGFR tyrosine kinase inhibitors. Lung cancer (Amsterdam, Netherlands). 2013; 82: 294-8.

27. Li W, Ren S, Li J, Li A, Fan L, Li X, et al. T790M mutation is associated with better efficacy of treatment beyond progression with EGFR-TKI in advanced NSCLC patients. Lung cancer (Amsterdam, Netherlands). 2014; 84: 295-300.

28. Meador CB, Lovly CM. Liquid biopsies reveal the dynamic nature of resistance mechanisms in solid tumors. Nature medicine. 2015; 21: 663-5.

29. Karlovich C, Goldman JW, Sun JM, Mann E, Sequist LV, Konopa K, et al. Assessment of EGFR Mutation Status in Matched Plasma and Tumor Tissue of NSCLC Patients from a Phase I Study of Rociletinib (CO-1686). Clinical cancer research: an official journal of the American Association for Cancer Research. 2016; 22: 2386-95.

30. Seki Y, Fujiwara Y, Kohno T, Takai E, Sunami K, Goto Y, et al. Picoliter-Droplet Digital Polymerase Chain Reaction-Based Analysis of Cell-Free Plasma DNA to Assess EGFR Mutations in Lung Adenocarcinoma That Confer Resistance to Tyrosine-Kinase Inhibitors. The oncologist. 2016; 21: 156-64.

31. Ishii H, Azuma K, Sakai K, Kawahara A, Yamada K, Tokito T, et al. Digital PCR analysis of plasma cell-free DNA for non-invasive detection of drug resistance mechanisms in EGFR mutant NSCLC: Correlation with paired tumor samples. Oncotarget. 2015; 6: 30850-8.

32. Sueoka-Aragane N, Katakami N, Satouchi M, Yokota S, Aoe K, Iwanaga K, et al. Monitoring EGFR T790M with plasma DNA from lung cancer patients in a prospective observational study. Cancer science. 2016; 107: 162-7.

33. Ilie M, Hofman V, Long E, Bordone O, Selva E, Washetine K, et al. Current challenges for detection of circulating tumor cells and cell-free circulating nucleic acids, and their characterization in non-small cell lung carcinoma patients. What is the best blood substrate for personalized medicine? Annals of translational medicine. 2014; 2: 107.

34. Hur JY, Kim HJ, Choi C-M, Lee JC, Lee KY. Liquid biopsy using extracellular vesicular DNA for EGFR mutation testing in the patients with NSCLC. AACR; 2017.

35. Oxnard GR, Thress KS, Alden RS, Lawrance R, Paweletz CP, Cantarini M, et al. Association Between Plasma Genotyping and Outcomes of Treatment With Osimertinib (AZD9291) in Advanced Non-Small-Cell Lung Cancer. Journal of clinical oncology: official journal of the American Society of Clinical Oncology. 2016; 34: 3375-82.

36. Matsuo N, Azuma K, Sakai K, Hattori S, Kawahara A, Ishii H, et al. Association of EGFR Exon 19 Deletion and EGFR-TKI Treatment Duration with Frequency of T790M Mutation in EGFR-Mutant Lung Cancer Patients. Sci Rep. 2016; 6: 36458.

37. Kuiper JL, Heideman DA, Thunnissen E, Paul MA, van Wijk AW, Postmus PE, et al. Incidence of T790M mutation in (sequential) rebiopsies in EGFR-mutated NSCLC-patients. Lung cancer (Amsterdam, Netherlands). 2014; 85: 19-24.

38. Zou B, Lee VHF, Chen L, Ma L, Wang DD, Yan H. Deciphering mechanisms of acquired T790M mutation after EGFR inhibitors for NSCLC by computational simulations. Scientific reports. 2017; 7: 6595. 